\title{
LA MEDITACIÓN EN LA ESPERANZA EN EL PROGRAMA EBC: DESCRIPCIÓN Y MECANISMOS DE ACCIÓN IMPLICADOS.
}

\section{THE HOPE MEDITATION IN CBWT PROGRAM: DESCRIPTION AND MECHANISMS OF ACTION}

\author{
David Alvear Morón \\ Departamento de Psicología Evolutiva y de la Educación. \\ Universidad del País Vasco/Euskal Herriko Unibertsitatea. España \\ ORCID: https://orcid.org/0000-0001-7299-1729
}

Cómo referenciar este artículo/How to reference this article:

Alvear, D. (2020). La Meditación en la Esperanza en el Programa EBC: Descripción y Mecanismos de Acción Implicados. Revista de Psicoterapia, 31(117), 43-56. https://doi.org/10.33898/rdp. v31i117.447

\section{Resumen}

Se describe el modelo de bienestar del programa EBC (Entrenamiento en Bienestar basado en prácticas Contemplativas) fundamentado en la acción virtuosa $y$ en la felicidad ego-desprendida, donde el bienestar subjetivo es un subproducto y un reforzador derivado de las propias acciones virtuosas. Posteriormente, se describe y se analizan los mecanismos de acción implícitos en la meditación en la esperanza, entendida como una meditación estratégica en el desarrollo del programa EBC. Para finalizar, se proponen varias conclusiones al respecto.

Palabras clave: meditación, bienestar psicológico, ciencias contemplativas, psicología positiva, esperanza.

\begin{abstract}
The wellbeing model of the CBWT program (Contemplative-Based Wellbeing-Training) is described. This program's wellbeing model is based on virtuous action and selflessness happiness, where subjective well-being is a by-product and a reinforcer derived from virtuous actions themselves. Subsequently, the hope meditation, understood as a strategic meditation in the development of the CBWT program, is described and the mechanisms of action which are implicit in this practice are analyzed. Finally, several conclusions are proposed in this regard.
\end{abstract}

Keywords: meditation, psychological well-being, contemplative sciences, positive psychology, hope. 
The Present Is Mother of the Future.

--Maha Ghosananda. Step by step

El bienestar entendido como un concepto integral y abarcador se puede definir como un estado de funcionamiento exitoso a lo largo de la vida que integra la función física, cognitiva y socioemocional (Pollard y Davidson, 2001). Los modelos psicológicos tradicionales, que tenían como eje el bienestar, han sido más bien escasos, si bien la gran mayoría de ellos han entendido el bienestar como un constructo multidimensional en el que se combinaban aspectos hedónicos y eudaimónicos (Huppert y So, 2013; Vázquez y Hervás, 2008).

El entrenamiento en bienestar basado en prácticas contemplativas (EBC) es un programa de intervención de 8 módulos sustentado en un marco teórico denominado como psicología positiva contemplativa (Cebolla y Alvear, 2019; Cebolla et al., 2019). La psicología positiva contemplativa es el área de la psicología positiva que incluye un abanico de técnicas y conceptualizaciones desarrolladas por las ciencias contemplativas para el fomento del bienestar, a partir de estrategias basadas en la evidencia (Alvear, 2015, 2018; Cebolla et al., 2017; Cebolla y Alvear, 2019). La premisa de la que se parte en el programa EBC es que, a través del entrenamiento mental que supone la práctica de la meditación, se pueden entrenar las competencias y habilidades necesarias que facilitan alcanzar una mente equilibrada y virtuosa con el objetivo de transformar la perspectiva del self, al pasar del egocentrismo a un ego desprendido. Para ello, el EBC se fundamenta en varios modelos teóricos de los que destacan dos: a) el modelo de actividad eudaimónica de Ken Sheldon (2013, 2018), y b) el modelo de la felicidad ego-céntrica/desprendida de Michaël Dambrun y Matthieu Ricard (2011). Para operativizar, articular, e implemetar esta propuesta en una intervención manualizada que entrene habilidades psicológicas básicas se utilizó la propuesta de los cuatros constituyentes de Richard J. Davidson (Davidson y Schuyler, 2015) y un quinto constituyente sobre la multimplicidad del self (Singer y Engert, 2019; McConnell, 2011). El nombre y los temas tratados en las 8 sesiones que componen los módulos del EBC se puede observar en la tabla 1.

\section{El Bienestar Subjetivo como Subproducto y Reforzador Derivado de Acciones Virtuosas}

En la historia de la filosofía, las teorías que fundamentan la felicidad en el placer (hedonismo) y aquellas que lo hacen basándose en una vida virtuosa (eudaimonía) han estado enfrentadas. Si bien la psicología científica de los últimos lustros ha realizado numerosos intentos para integrarlas, esta tarea no ha estado exenta de discrepancias, falta de consenso y cierta confusión (Huta y Waterman, 2014). El programa EBC se centra en el modelo de actividad eudaimónica de Sheldon $(2013,2018)$, y, por lo tanto, defiende la eudaimonía como un término concebido originalmente como una vía de actividad virtuosa, subrayando este aspecto de acción o actividad, y no tanto como un sentimiento, como una condición psicológica 
Tabla 1. Guion y Objetivos de cada Sesión del Programa EBC

\begin{tabular}{|c|c|c|}
\hline & Nombre de la sesión & Temas tratados \\
\hline 1 & $\begin{array}{l}\text { Motivación y orientación } \\
\text { a la felicidad }\end{array}$ & $\begin{array}{l}\text { Introducir el curso y grupo } \\
\text { Describir las normas } \\
\text { Explicar los dos modelos sobre los que se apoya el } \\
\text { entrenamiento } \\
\text { Preparando la motivación } \\
\text { Introducción a las prácticas imaginativas } \\
\end{array}$ \\
\hline 2 & $\begin{array}{l}\text { Mindfulness o la } \\
\text { importancia de la } \\
\text { atención }\end{array}$ & $\begin{array}{l}\text { Introducción al primer constituyente: mindfulness } \\
\text { Los obstáculos de la práctica }\end{array}$ \\
\hline 3 & $\begin{array}{l}\text { Potenciando las } \\
\text { emociones positivas }\end{array}$ & $\begin{array}{l}\text { Conocimiento de las emociones positivas y de su } \\
\text { clasificación. } \\
\text { Adquisición y entrenamiento de la habilidad del saboreo } \\
\text { de experiencias agradables del pasado, del presente y } \\
\text { del futuro } \\
\text { Conocimiento y práctica de una emoción positiva: la } \\
\text { gratitud }\end{array}$ \\
\hline 4 & $\begin{array}{l}\text { Orientar la mente hacia } \\
\text { las fortalezas }\end{array}$ & $\begin{array}{l}\text { Conocimiento de las fortalezas personales y de su } \\
\text { clasificación, para detectarlas con una mayor facilidad } \\
\text { en la cotidianeidad } \\
\text { Detectar las cinco fortalezas principales en uno mismo y } \\
\text { las fortalezas principales del grupo } \\
\text { Aprender a llevar a cabo conductas y actividades } \\
\text { vinculadas a fortalezas personales }\end{array}$ \\
\hline 5 & $\begin{array}{l}\text { Recuperarnos de la } \\
\text { adversidad: regulando } \\
\text { emociones negativas }\end{array}$ & $\begin{array}{l}\text { Introducción al tercer constituyente: resiliencia o } \\
\text { recuperación frente a la adversidad } \\
\text { Aprender el papel de las emociones negativas para el } \\
\text { bienestar psicológico } \\
\text { Entrenamiento de habilidades psicológicas para } \\
\text { mantener el bienestar, incluso en momentos de } \\
\text { adversidad } \\
\text { Introducción a la autocompasión } \\
\text { Promover y entrenar estrategias de buen trato mediante } \\
\text { prácticas generativas }\end{array}$ \\
\hline 6 & $\begin{array}{l}\text { De la compasión a la } \\
\text { generosidad: la felicidad } \\
\text { a partir de la entrega }\end{array}$ & $\begin{array}{l}\text { Conocer la importancia de la generosidad, altruismo y } \\
\text { compasión para alcanzar el bienestar } \\
\text { Entrenar las técnicas que se han mostrado más } \\
\text { eficaces para su desarrollo }\end{array}$ \\
\hline 7 & $\begin{array}{l}\text { Dando voz a la } \\
\text { compasión: las diferentes } \\
\text { partes de tu mente }\end{array}$ & $\begin{array}{l}\text { Introducción a las partes (subpersonalidades) que } \\
\text { habitan la mente, para ganar perspectiva a la hora de } \\
\text { entender la mente } \\
\text { Aprender a detectar y cultivar la parte compasiva que } \\
\text { habita en la mente de cada ser humano } \\
\text { Aprender a cuidar las partes que habitan en la mente de } \\
\text { cada persona mediante la parte compasiva }\end{array}$ \\
\hline 8 & $\begin{array}{l}\text { El resto de tus días } \\
\text { vividos con sentido }\end{array}$ & $\begin{array}{l}\text { Cerrar el programa y obtener conclusiones para poder } \\
\text { continuar con la práctica }\end{array}$ \\
\hline
\end{tabular}


o como un tipo de bienestar.

En psicología, el bienestar subjetivo ha sido definido como el resultado combinado de tres componentes (Diener, 2000): niveles altos de afecto positivo, niveles bajos de afecto negativo y niveles altos de satisfacción con la vida. Detrás de esta definición, es decir, de la consecución de placer y la evitación del dolor, hay implícito un planteamiento vital hedonista que sostiene culturalmente el modelo de sociedad de consumo, e, incluso, el modelo de felicidad predominante en la actualidad (Cebolla y Alvear, 2019). En el modelo de bienestar defendido en EBC se propone que el bienestar subjetivo sea el resultado (el subproducto) de la acción virtuosa y, por ende, la acción o actividad virtuosa sería la causa de la felicidad (Sheldon, 2013, 2018). De esta manera, en este modelo se entiende que, cada persona, en cada momento, posee una amplia variedad de opciones basadas en qué hacer, con quién, qué creer o pensar, o a qué prestar atención. La ventaja de tomar decisiones sabias y virtuosas (acciones eudaimónicas) basadas en todas estas opciones deriva en experiencias satisfactorias. Y, finalmente, estas experiencias satisfactorias tienden a aumentar los niveles de bienestar subjetivo y refuerzan las decisiones sabias y virtuosas, motivando al sujeto a volverlas a realizar (Sheldon, 2018).

En el EBC se plantea que para el aumento de la eudaimonía es necesario el cultivo de un carácter virtuoso, depositando el foco principal en la actividad que refleje la virtud, la excelencia, lo mejor que hay dentro de cada uno nosotros y el total desarrollo de nuestro potencial (Tiberius y Hall, 2010). En conclusión, en este modelo, el placer de base hedonista o bienestar subjetivo se integra como un subproducto o un indicador de la búsqueda de la virtud. Derivado de esto, el programa EBC pretende entrenar predictores y actividades virtuosas como la esperanza, el saboreo y la compasión, entre otras muchas, para ayudar al usuario a aumentar el nivel de bienestar subjetivo que posteriormente derive en una manutención de la actividad virtuosa.

\section{Descripción de la Meditación en la Esperanza (ME)}

El programa EBC se compone de múltiples prácticas y actividades para aumentar la acción virtuosa: 21 ejercicios de auto-registro, 10 prácticas meditativas, un cuestionario, una dinámica grupal y una actividad externa a realizar en vida cotidiana. De todas ellas, destaca la Meditación en la Esperanza (ME) como la práctica contemplativa transversal más importante del entrenamiento.

La ME es una meditación de tipo generativo que pertenece a la familia de las meditaciones constructivas (Dahl et al., 2015). En la ME se llevan a cabo estrategias cognitivo-atencionales basadas en habilidades relativas a la regulación de la atención, imaginería, y cognición prospectiva (Cebolla y Alvear, 2019). Primeramente, se realiza en formato de meditación guiada mediante audio y, posteriormente, cuando el usuario haya memorizado las fases de la ME, la puede llevar a cabo sin apoyo del audio. Esta meditación guía a la persona a imaginarse un momento de felicidad/ eudaimonía evocando todos sus detalles y sensaciones, dirigiéndole posteriormen- 
te a reflexionar acerca de las fortalezas y habilidades que fueron necesarias para generar ese momento. Prosigue extendiendo esa visión de felicidad/eudaimonía en un año, en cinco años, en veinte años y finalmente hasta la vejez, haciéndose la misma pregunta, ¿qué fortalezas y prácticas tendría que desarrollar para llegar a ese momento de bienestar?

\section{Fases de la ME y Justificación Teórica en el Programa EBC}

Si desglosamos la ME se puede ver cómo se compone de seis fases, la grabación de audio tiene una duración de 19 minutos. En la fase 1, se realiza una unificación de la atención mediante meditación concentrativa. En fase 2, primeramente se evoca y posteriormente se sostiene una actividad mental de saboreo de una experiencia muy satisfactoria del pasado, a la vez, se detectan las posibles habilidades, fortalezas o virtudes que fueron causa (parcial, por supuesto) de la citada experiencia. Más adelante, en la fase 3, mediante la cognición prospectiva, se evoca y saborea una hipotética experiencia futura muy satisfactoria, en este caso en un futuro relativamente cercano (dentro de un año), junto a ello, tal y como se hizo en la fase 2, se detectan las habilidades, virtudes o fortalezas que sostienen y son causa (parcial) de la citada experiencia hipotética futura, y que son susceptibles de entrenar y aumentar en el presente. En las fases 4, 5, y 6 se lleva a cabo la misma actividad mental que en la fase 3 , con la diferencia que en la fase 4 el futuro al que se proyecta la experiencia muy satisfactoria es a dentro de cinco años, en la fase 5 es a dentro de veinte años, y, por último, en la fase 6 se proyecta a la vejez o ancianidad la citada experiencia muy satisfactoria. Si el usuario es una persona mayor, se puede suprimir la fase 5 y directamente pasar de la fase 4 a la fase 6 . En la tabla 2. se pueden observar las seis fases, la actividad mental generada y el tiempo orientativo recomendado para cada fase.

Tabla 2. Fases, Actividad Mental Generada y el Tiempo Orientativo Recomendado para cada Fase en la Meditación en la Esperanza (ME)

\begin{tabular}{lll}
\hline FASE ACTIVIDAD MENTAL & TIEMPO ORIENTATIVO \\
\hline 1 & Unificación de la atención en la respiración & 3 minutos \\
\hline 2 & $\begin{array}{l}\text { Evocar y saborear mediante imágenes una experiencia } \\
\text { muy satisfactoria del pasado. Detectar las habilidades, } \\
\text { virtudes o fortalezas que son causa (parcial) de esta }\end{array}$ & 4 minutos \\
& experiencia & \\
\hline $3 \quad$ & $\begin{array}{l}\text { Evocar y saborear mediante imágenes una experiencia } \\
\text { muy satisfactoria del futuro cercano (1 año). Detectar las } \\
\text { habilidades, virtudes o fortalezas que son causa (parcial) } \\
\text { de esta experiencia y que son susceptibles de entrenar } \\
\text { en el presente }\end{array}$ \\
\hline
\end{tabular}




\begin{tabular}{lll}
\hline FASE ACTIVIDAD MENTAL & TIEMPO ORIENTATIVO \\
\hline 4 & $\begin{array}{l}\text { Evocar y saborear mediante imágenes una experiencia } \\
\text { muy satisfactoria del futuro medio (5 años). Detectar las } \\
\text { habilidades, virtudes o fortalezas que son causa (parcial) } \\
\text { de esta experiencia y que son susceptibles de entrenar } \\
\text { en el presente }\end{array}$ \\
\hline $5 \quad$ & $\begin{array}{ll}\text { Evocar y saborear mediante imágenes una experiencia } \\
\text { muy satisfactoria del futuro lejano (20 años). Detectar las }\end{array}$ \\
& $\begin{array}{l}\text { habilidades, virtudes o fortalezas que son causa (parcial) } \\
\text { de esta experiencia y que son susceptibles de entrenar }\end{array}$ \\
& en el presente \\
\hline $6 \quad$ & $\begin{array}{l}\text { Evocar y saborear mediante imágenes una experiencia } 2 \text { minutos } \\
\text { muy satisfactoria del futuro muy lejano (vejezlancianidad). }\end{array}$ \\
& $\begin{array}{l}\text { Detectar las habilidades, virtudes o fortalezas que } \\
\text { son causa (parcial) de esta experiencia y que son } \\
\text { susceptibles de entrenar en el presente }\end{array}$ \\
\hline
\end{tabular}

La ME se encuentra integrada en el núcleo pedagógico del plan de trabajo para aumentar el bienestar propuesto en el EBC. El objetivo es que la actividad elaborada a la hora de crear en la mente un potencial yo-futuro con la mejor versión de uno/a mismo/a no se quede exclusivamente en un ansia o anhelo, sino que se desarrolle un plan de implementación. Por consiguiente, se dedica un tiempo a aclarar y planificar qué aspectos del participante (fortalezas, habilidades, actitudes, etc.) cree que debe desarrollar para alcanzar el bienestar. Este proceso de aclarar y planificar un yo-futuro cercano a la excelencia se desarrolla mediante el escrito y constante supervisión a lo largo de todo el entrenamiento de una carta. La carta se redacta en la primera semana del programa, a partir del conocimiento previo que posee el usuario, y se va modificando a lo largo del entrenamiento en la medida en que se van entrenando nuevas habilidades y se va ampliando el lenguaje y el léxico sobre las fortalezas personales en particular y sobre el bienestar en general.

\section{Comparativa de la ME con el Ejercicio del Mejor Yo Posible (Best possible self-BPS)}

En la ME se trata de combinar el potencial del ejercicio del Mejor yo posible proveniente de la psicología positiva con aspectos de tipo contemplativo centrados en la generación de imágenes y estados mentales. El ejercicio del mejor yo posible (Best Possible Self-BPS) es una intervención de psicología positiva orientado al futuro que se ha mostrado eficaz a la hora de incrementar el nivel de bienestar y del estado de ánimo (Enrique et al., 2018; Meevissen et al., 2011; Peters et al., 2010; 
Renner et al., 2014), y en el aumento del optimismo (Malouff y Schutte, 2016). Este ejercicio fue inicialmente propuesto por King (2001) como ejercicio de escritura orientado a promover procesos de auto-regulación, pero sin la carga emocional negativa que implicaban los ejercicios anteriormente empleados como la escritura de acontecimientos traumáticos (Pennebaker, 1997). El BPS se considera una técnica de pensamiento futuro positivo, que consiste en la visualización y escritura de un futuro en el que su vida se ha desarrollado de la forma que desean, cumpliendo todas sus metas y objetivos planteados (King, 2001). Desde entonces, más de 30 estudios han aplicado este ejercicio y evaluado sus efectos (Loveday et al., 2018). En el ejercicio del BPS los participantes visualizan y escriben sobre el "mejor yo posible” en el futuro siguiendo instrucciones claras y sencillas, un ejemplo de las consignas pautadas en varias investigaciones es el siguiente:

Has sido asignado al azar para participar en una condición en la que vas a pensar sobre tu "mejor yo posible". Con este posible mejor "yo" queremos que te imagines a ti mismo/a en un futuro en el que todo se ha desarrollado de la mejor manera posible. Te has esforzado mucho y has conseguido alcanzar todos los objetivos que te habías planteado en tu vida. Puedes imaginártelo como el hecho de haber conseguido todos tus sueños y de haber desarrollado todas tus potencialidades.

Dentro de un momento, te voy a pedir que pienses en la mejor manera posible en que tu vida podría desarrollarse en 4 ámbitos (personal, social, profesional y de la salud), con el objetivo de dirigir las decisiones que estás tomando en la actualidad.

Para poder determinar y guiar la construcción de tu mejor posible "yo", durante los próximos 25 minutos, vas a pensar y a escribir las metas, habilidades y deseos que te gustaría adquirir en un futuro lejano dentro de cada uno de los cuatro ámbitos que hemos mencionado. Tienes que unificarlos en una especie de diario personal. A lo largo de este proceso, piensa en habilidades realistas y en metas/deseos que sean posibles de alcanzar y que te gustaría adquirir o lograr en el futuro. (Enrique, 2017, p. 217)

Las limitaciones encontradas en las investigaciones basadas en BPS tienen que ver con dos cuestiones. Por un lado, parece que las intervenciones basadas en objetivos supera a aquellas intervenciones orientadas a mejorar el optimismo como el BPS, las intervenciones basadas en objetivos engloban no solo el planteamiento de metas y expectativas de resultado sino también la planificación y el desarrollo de pasos dirigidos a la consecución de los propios objetivos (Enrique, 2017). Una explicación plausible al respecto podría deberse a que el ejercicio del BPS carece del planteamiento de las intenciones de implementación (Schweiger Gallo et al., 2007) y que podría generar una falsa esperanza, definida por Kwon (2002) como el estado en el que se tiene una meta deseada y motivación para alcanzarla, pero se carece de los planes para llegar a ella. Por otro lado, otra limitación tendría que ver con que el BSP no se encuentra enraizado en un modelo teórico que explique 
su eficacia (Saiz, 2018).

La falta de intenciones de implementación en el ejercicio del BPS se intenta paliar en la Meditación en la esperanza mediante el uso de fortalezas y habilidades personales (Park y Peterson, 2009; Peterson y Seligman, 2004) como elementos de implementación. Por ejemplo, en muestra con sujetos adolescentes se ha visto que, según la teoría de la motivación basada en la identidad, una forma de aumentar las probabilidades de que el alumnado esté motivado consiste en que su yo-futuro adulto se encuentre conectado con su yo-actual (Nurra y Oyserman, 2018). En el caso del programa EBC se intenta, mediante diversas estrategias, que las fortalezas personales actúen como puente entre el presente y la meta deseada.

Por otro lado, el ejercicio del BPS es una técnica en la que se implementa la visualización (Meevissen et al., 2011), mas no se entrena al sujeto de manera explícita en cómo visualizar, ni siquiera se ofrecen pautas o instrucciones concretas para que pueda visualizar y sostener imágenes en la mente de una forma más efectiva. Este proceso de atención y mantenimiento de la imagen mental podría verse favorecido al incorporar ciertas características de la práctica de la meditación mindfulness. Se considera que el mindfulness y, más concretamente la práctica meditativa concentrativa, conlleva atender de forma intencionada a un objeto (como la respiración, el cuerpo, los sonidos, una imagen mental...), observando las sensaciones, pensamientos y emociones que emergen en ese momento (Vago y Silbersweig, 2012). Por tanto, y tal y cómo exponen Cebolla et al. (2017), la meditación mindfulness puede actuar como catalizador de las intervenciones positivas al atender y auto-observar la experiencia interna con conciencia plena. Junto a los beneficios que puede aportar el propio entrenamiento en mindfulness, en el programa EBC se destinan dos ejercicios a aprender a visualizar de manera más efectiva basados en el trabajo de Emily Holmes y su equipo (Pearson et al. 2013) (ver subapartado sobre habilidad imaginativa optimizada).

Hasta la fecha solo existe un estudio aleatorizado en el que se comparaba la eficacia de la ME frente al BPS en afecto positivo y negativo, autocompasión, bienestar psicológico, expectativas futuras, optimismo y esperanza (Saiz, 2018). Se observó que la ME produjo cambios significativos en afecto negativo, expectativas futuras negativas, autoaceptación, autonomía y propósito en la vida, pero estos cambios no fueron significativamente mayores que los producidos por BPS. También se detectó que la autocompasión moderaba el efecto de la intervención, siendo las personas menos autocompasivas las que más se benefician de la ME (Saiz, 2018). De este trabajo se deduce que la ME es una intervención basada en psicología positiva válida, y aceptada y valorada positivamente por los participantes. De la misma manera, este trabajo también detectó que la ME requiere de un entrenamiento previo en mindfulness para potenciar sus efectos. 


\section{Propuesta de los Mecanismos de Acción Implicados en la ME}

En la fase actual del programa EBC no es posible confirmar con certeza cuales pueden ser los mecanismos de acción implicados en la ME. No obstante, y partiendo de las premisas teórico-científicas con las que se diseñó la ME, podemos realizar un acercamiento hipotético a los posibles mecanismos de acción implicados en esta práctica meditativa. Partimos de cuatro estrategias implícitas que pueden ayudar a implementar el bienestar en los sujetos que practiquen la ME: el saboreo del pasado y futuro, la cognición prospectiva, el aprendizaje por modelado y la visualización optimizada.

\section{Saboreo del Pasado y del Futuro}

El saboreo (savoring) es una técnica desarrollada por Fred Bryant, que implica la habilidad de intensificar y prolongar los sentimientos positivos de forma deliberada, incrementando la toma de consciencia (awareness) y la apreciación de las experiencias positivas (Bryant y Veroff, 2007). Al igual que sucede con mindfulness, la capacidad de saboreo difiere de una persona a otra, y es susceptible de ser entrenada, ya que tiene un componente rasgo que es temperamental y un componente estado fruto de la práctica concreta. Cuando se practica el saboreo se enfoca la atención en las experiencias positivas mientras se interviene para modificar los pensamientos y las conductas, de manera que se intensifiquen y prolonguen los sentimientos positivos (Bryant et al., 2011). A nivel neurocientífico, se ha detectado que los correlatos neurales del saboreo están asociados con una activación sostenida del estriado ventral y con una conectividad sostenida entre la corteza prefrontal y el estriado ventral (Heller et al., 2013). Desde una dimensión temporal se han explorado tres dimensiones: el saboreo centrado en el futuro, el saboreo mediante la rememoración del pasado y el saboreo del momento presente (Bryant, 2003). Partiendo de estas dimensiones se han elaborado tres tipos de intervenciones basadas en saboreo: a) Las intervenciones para aprender a saborear el pasado; por ejemplo, pensar sobre sucesos biográficos positivos del pasado o la reminiscencia positiva; b) Las intervenciones para aprender a saborear el presente, como el incremento de estrategias para saborear la cotidianidad o entrenar un enfoque atencional positivo; c) Las intervenciones basadas en el saboreo del futuro.

En el caso de la ME, en la fase 2 se llevan a cabo micro-experiencias de visualización orientadas a saborear el pasado, lo que técnicamente toma el nombre de reminiscencia positiva, en las que se generan imágenes mentales (y también se re-experimenta corporalmente) relativas a un suceso positivo del pasado. En las fases 3, 4, 5 y 6 se generan imágenes mentales correspondientes a un yo-futuro con un nivel alto de excelencia y virtuoso, y, por ende, podríamos clasificarlo como un saboreo del futuro (Cebolla y Alvear, 2019). 


\section{Cognición Prospectiva}

Tal y como se vio en el subapartado anterior desde un punto de vista afectivo, cuando en la ME se evoca un yo-futuro virtuoso se suele hacer insertado en una actividad de saboreo del futuro. No obstante, la perspectiva de futuro no implica solamente este aspecto afectivo, a la vez, la mente está generando una estrategia relativa a la regulación de la atención dirigiéndola al futuro, más exactamente, estamos hablando de cognición prospectiva (MacLeod et al., 1997).

En la ME se combinan tres aspectos: la capacidad cognitivo-prospectiva a orientar la mente a un yo-futuro, la base afectiva que supone el hecho de que este yo-futuro sea potencialmente compasivo y virtuoso, y la motivación para cuidar y hacerse cargo desde el yo-presente de este yo-futuro imaginado. En este sentido, se pretende que este yo-futuro se encuentre alineado con el yo-presente en forma de potenciales fortalezas personales que se puedan activar/entrenar en la actualidad (Nurra y Oyserman, 2018).

En relación al cuidado del yo-futuro nos basamos en dos trabajos. En los primeros se evaluó el impacto de vernos como ancianos a la hora de tomar decisiones para planificar nuestras finanzas tras el retiro laboral. Los resultados del estudio mostraron que aquellas personas que sintieron empatía por esa versión de sí mismos ancianos invirtieron más dinero (es decir, mostraron mayor autocontrol) para ahorrar, perdiendo la posibilidad de gastarlo en alguna actividad hedónica en el presente (Hershfield, 2011). En un segundo trabajo, en una dirección inversa, se invitó a un grupo de participantes a que interactuaran con una versión vívida y realista de sí mismos en el futuro, y posteriormente llevaron a cabo una tarea experimental en la que, para recibir una recompensa económica, había que falsear y mentir. Los resultados mostraron que el grupo que interactuó con ese yo futuro engañaba menos en la prueba que el grupo de control (Van Gelder et al., 2013).

La familiarización e identificación con un yo-futuro es un elemento clave en la ME, en muchas ocasiones se trata al yo-futuro como si fuera otra persona (Hershfield, 2011; Van Gelder et al., 2013), es decir, no hay una alta identificación o incluso la persona se muestra ajena respecto a su yo-futuro. Este hecho, obviamente, va a determinar los procesos de toma de decisiones, estrategias de autocontrol, compromiso y conexión con valores del presente.

\section{Aprendizaje por Modelos Basado en Ideal del yo Futuro}

El modelo ideal del yo ha sido ampliamente tratado en la literatura científica (Boyatzis y Akrivou, 2006; Wille et al., 2018). Tanto en el programa EBC, como específicamente en la ME, se procura ofrecer instrucciones al usuario para que genere un modelo ideal del yo adaptado a su contexto y que, a la vez, suponga un elemento motivacional para poder modificar su conducta ayudado por el desarrollo de fortalezas personales.

El aprendizaje por modelos, o modelado, se suele definir como el aprendizaje que se adquiere por medio de la observación del comportamiento de otra persona 
(Barraca, 2014). En el caso de la ME, se lleva a cabo un modelado encubierto, basado también en aprendizaje vicario, aunque en este caso el proceso de modelado se sigue de forma encubierta (mediante la imaginación) (Cautela, 1971; Kazdin, 1978) al estar fundamentado en modelos de imitación de un yo-futuro virtuoso generado por imágenes mentales.

$\mathrm{Al}$ entrenar de manera combinada la escritura/lectura de la carta del modelo ideal del yo-futuro junto a la ME, se realiza un acercamiento paulatino a un modelo ideal de un yo-futuro virtuoso que se va depurando semana a semana en el propio entrenamiento.

\section{Habilidad Imaginativa Optimizada}

Tal como se señaló, la ME se cataloga como una práctica meditativa constructiva o generativa. La familia de las prácticas constructivas suele requerir del uso de la imaginación para inducir estados afectivos positivos, compasión, ecuanimidad, autocompasión, etc. Estas imágenes mentales generadas funcionarían como una especie de proceso de inducción de estados mentales positivos o virtuosos; por ello, las habilidades imaginativas son clave para su desarrollo y eficacia (Cebolla y Alvear, 2019).

En la actualidad es conocido que las imágenes mentales poseen la capacidad de evocar una respuesta emocional, hasta el punto de que se activan las mismas áreas cerebrales tanto al imaginar como al ver la misma imagen (Ji et al., 2016). En la ME se le da un gran valor al hecho de ofrecer instrucciones claras y basadas en la evidencia con el objetivo de conseguir una capacidad de imaginación eficaz. Para esta tarea nos basamos en los cuatro mecanismos esenciales en el proceso de imaginar propuestos por Holmes y su equipo (Pearson et al., 2013): la creación de la imagen mental, la capacidad de sostener la imagen mental, la capacidad de generar viveza y definición en la imagen mental, y la rotación y transformación de la imagen mental. A estos cuatro mecanismos le añadimos un quinto, relacionado con la incorporación del resto de sentidos sensoriales durante el proceso de imaginación.

Cualesquiera que sean las habilidades imaginativas que posea el practicante, tras el entrenamiento la práctica irá mejorando los cinco mecanismos, entre ellos el de el sostén y la posibilidad de transformación de la imagen mental. Debido a ello, es fundamental haber trabajado la práctica de la meditación concentrativa en entrenamientos anteriores, esta capacidad previa ayudará al practicante a mantener la imagen mental construida y a gestionar la divagación mental que podría obstaculizar la inducción de la experiencia imaginativa.

\section{Conclusiones}

La ME es una práctica meditativa de tipo generativo integrada en el entrenamiento en bienestar basado en prácticas contemplativas (programa EBC). Puede resultar de utilidad para integrarlo en un proceso psicoterapéutico con usuarios que hayan entrenado previamente habilidades mindfulness con el objetivo de aumentar 
el bienestar y los niveles de esperanza rasgo.

La presentación de la ME en este escrito es un acercamiento teórico y descriptivo. Se necesitan estudios futuros que comparen la eficacia de la ME con otro tipo de técnicas, tanto con las relacionadas con aspectos prospectivos de un yo-futuro optimizado (e.g. BPS) como con prácticas meditativas generativas (e.g. meditación en compasión). También son necesarios trabajos que confirmen o descarten los cinco mecanismos de acción implicados propuestos.

\section{Referencias}

Alvear, D. (2015). Mindfulness en positivo. La ciencia de la atención plena y la psicología positiva en el camino del bienestar. Milenio.

Alvear, D. (2018). Mindfulness, psicología positiva e inteligencia emocional. En M. A. Santed y S. Segovia (coords.), Fundamentos y aplicaciones. (pp. 185-206). Paraninfo Universidad.

Barraca, J. (2014). Técnicas de modificación de conducta: una guía para su puesta en práctica. Síntesis.

Boyatzis, R. y Akrivou, K. (2006). "The ideal self as the driver of intentional change”. Journal of Management Development, 25(7), 624-642. https://doi.org/10.1108/02621710610678454

Bryant, F. B. (2003). Savoring beliefs inventory (SBI): A scale for measuring beliefs about savouring. Journal of Mental Health, 12(2), 175-196. https://doi.org/10.1080/0963823031000103489

Bryant, F., Chadwick, E. y Kluwe, K. (2011). Understanding the processes that regulate positive emotional experience: unsolved problems and future directions for theory and research on savoring. International Journal of Wellbeing, 1(1), 107-126. https://doi.org/10.5502/ijw.v1i1.18

Bryant, F. B. y Veroff, J. (2007). Savoring: A new model of positive experience. Lawrence.

Cautela, J. R. (1971). Covert conditioning. En A. Jacobs y L. B. Sachs (eds.), The psychology of private events: Perspectives on covert response systems (pp. 109-130). Academic Press.

Cebolla, A. y Alvear, D. (2019). Psicología positiva contemplativa. Fundamentos para un entrenamiento en bienestar basado en prácticas contemplativas (EBC). Kairós.

Cebolla, A., Enrique, A., Alvear, D., Soler, J. y García-Campayo, J. (2017). Contemplative positive psychology: introducing mindfulness into positive psychology. Psychologist Papers, 38(1), 12-18. https://doi. org/10.23923/pap.psicol2017.2816

Cebolla, A., Herrero, R., Carrillo, A., Navarro, J., Soler, J. y Alvear, D. (2019). Contemplative Practice-Based Well-Being Training (CBWT): Towards a Contemplative Positive Psychology. En L. Galiana y N. Sansó (eds.), The power of compassion (pp. 159-174). Nova Science Publishers.

Dahl, C. J., Lutz, A. y Davidson, R. J. (2015). Reconstructing and decons- tructing the self: Cognitive mechanisms in meditation practice. Trends in Cognitive Sciences, 19(9), 515-523. https://doi.org/10.1016/j.tics.2015.07.001

Dambrun, M. y Ricard, M. (2011). Self-centeredness and selflessness: a theory of self-based psychological functioning and its consequences for happiness. Review of General Psychology, 15, 138-157. https://doi. org/10.1037\%2Fa0023059

Davidson, R. y Schuyler, B. (2015). Neuroscience of happiness. En J. Helliwell, R. Layard y J. Sachs (eds.), World happiness report 2015 (pp. 88-105). Sustainable Development Solutions Network.

Diener, E. (2000). Subjective well-being: The science of happiness and a proposal for a national index. American Psychologist, 55(1), 34-43. https://doi.org/10.1037/0003-066X.55.1.34

Enrique, A. (2017). Efficacy of positive psychological interventions oriented toward the future: experimental studies with general and clinical populations. [Tesis doctoral. Universitat Jaume I (España)]. http://dx.doi. org/10.6035/14023.2017.209666

Enrique, Á., Bretón-López, J., Molinari, G., Baños, R. M. y Botella, C. (2018). Efficacy of an adaptation of the Best Possible Self intervention implemented through positive technology: A randomized control trial. Applied Research in Quality of Life, 13(3), 671-689. https://doi.org/10.1007/s11482-017-9552-5

Heller, A. S., van Reekum, C. M., Schaefer, S. M., Lapate, R. C., Radler, B. T., Ryff, C. D. y Davidson, R. J. (2013). Sustained ventral striatal activity predicts eudaimonic well-being and cortisol output. Psychological Science, 24(11), 2191-2200. https://doi.org/10.1177\%2F0956797613490744 
Hershfield, H. E. (2011). Future self-continuity: how conceptions of the future self transform intertemporal choice. Annals of the New York Academy of Sciences, 1235, 30-43. https://dx.doi.org/10.1111\%2Fj.17496632.2011.06201.x

Huppert, F. A. y So, T. C. (2013). Flourishing across Europe: Application of a new conceptual framework for defining well-being. Social Indicators Research, 110(3), 837-861.http://dx.doi.org/10.1007/s11205-011-9966-7

Huta, V. y Waterman, A. S. (2014). Eudaimonia and its distinction from hedonia: Developing a classification and terminology for understanding conceptual and operational definitions. Journal of Happiness Studies, 15(6), 1425-1456. https://doi.org/10.1007/s10902-013-9485-0

Ji, J. L., Heyes, S. B., MacLeod, C. y Holmes, E. A. (2016). Emotional mental imagery as simulation of reality: fear and beyond- a tribute to Peter Lang. Behaviour Therapy, 47(5), 702-719. https://doi.org/10.1016/j. beth.2015.11.004

Kazdin, A. E. (1978). Covert Modeling: The Therapeutic Application of Imagined Rehearsal. En J. L. Singer y K. S. Pope (eds.), The Power of Human Imagination. Emotions, Personality, and Psychotherapy (pp. 255-278). Springer. https://doi.org/10.1007/978-1-4613-3941-0_9

King, L. A. (2001). The health benefits of writing about life goals. Personality and Social Psychology Bulletin, 27(7), 798-807. https://doi.org/10.1177/0146167201277003

Kwon, P. (2002). Hope, defense mechanisms, and adjustment: implications for false hope and defensive hopelessness. Journal of personality, 70(2), 207-231. https://doi.org/10.1111/1467-6494.05003

Loveday, P. M., Lovell, G. P. y Jones, C. M. (2018). The Best Possible Selves Intervention: A Review of the Literature to Evaluate Efficacy and Guide Future Research. Journal of Happiness Studies, 19(2), 607-628. https://doi.org/10.1007/s10902-016-9824-z

MacLeod, A. K., Tata, P., Kentish, J. y Jacobsen, H. (1997). Retrospective and prospective cognitions in anxiety and depression. Cognition and Emotion, 11, 467-479. https://doi.org/10.1080/026999397379881

Malouff, J. M. y Schutte, N. S. (2017). Can psychological interventions increase optimism? A meta-analysis. The Journal of Positive Psychology, 12(6), 594-604. https://doi.org/10.1080/17439760.2016.1221122

McConnell, A. R. (2011). The multiple self-aspects framework: self-concept representation and its implications. Personality and Social Psychology Review, 15, 3-27. https://doi.org/10.1177\%2F1088868310371101

Meevissen, Y. M., Peters, M. L. y Alberts, H. J. (2011). Become more optimistic by imagining a best possible self: Effects of a two week intervention. Journal of Behavior Therapy and Experimental Psychiatry, 42(3), 371-378. https://doi.org/10.1016/j.jbtep.2011.02.012

Nurra, C. y Oyserman, D. (2018). From future self to current action: An identity-based motivation perspective. Self and Identity, 17(3), 343-364. https://doi.org/10.1080/15298868.2017.1375003

Park, N. y Peterson, C. (2009). Character strengths: research and practice. Journal of College and Character, 10(4). https://doi.org/10.2202/1940-1639.1042

Pearson, D. G., Deeprose, C., Wallace-Hadrill, S. M., Heyes, S. B. y Holmes, E. A. (2013). Assessing mental imagery in clinical psychology: A review of imagery measures and a guiding framework. Clinical Psychology Review, 33(1), 1-23. https://doi.org/10.1016/j.cpr.2012.09.001

Pennebaker, J. W. (1997). Writing about emotional experiences as a therapeutic process. Psychological Science, 8(3), 162-166. https://doi.org/10.1111\%2Fj.1467-9280.1997.tb00403.x

Peters, M. L., Flink, I. K., Boersma, K. y Linton, S. J. (2010). Manipulating optimism: Can imagining a best possible self be used to increase positive future expectancies? The Journal of Positive Psychology, 5(3), 204-211. https://doi.org/10.1080/17439761003790963

Peterson, C. y Seligman, M. E. P. (2004). Character strengths and virtues: A handbook and classification. American Psychological Association.

Pollard, E. L. y Davidson, L. (2001). Action research in family and early childhood: Foundations for child wellbeing. UNESCO Education Sector.

Renner, F., Schwarz, P., Peters, M. L. y Huibers, M. J. (2014). Effects of a best-possible-self mental imagery exercise on mood and dysfunctional attitudes. Psychiatry Research, 215(1), 105-110. https://doi.org/10.1016/j. psychres.2013.10.033

Saiz, I. (2018). Intervenciones psicológicas positivas: Eficacia de la Meditación en la Esperanza vs. El mejor yo posible [Trabajo de fin de Máster. Universitat de València. España].

Schweiger Gallo, I. y Gollwitzer, P. M. (2007). Implementation intentions: Control of fear despite cognitive load. Psicothema, 19, 280-285.

Sheldon, K. M. (2013). Individual daimon, universal needs, and subjective well-being: Happiness as the natural consequence of a life well lived. En A. Waterman (ed.), The best within us: Positive psychology perspectives on eudaimonic functioning (pp. 119-137). American Psychological Association. 
Sheldon, K. M. (2018). Understanding the good life: Eudaimonic living involves well-doing, not well-being. En J. P. Forgas y R. E: Baumeister (eds.), The Social Psychology of Living Well (pp. 116-136). Routledge.

Singer, T. y Engert, V. (2019). It matters what you practice: differential training effects on subjective experience, behavior, brain and body in the ReSource Project. Current Opinion in Psychology, 28, 151-158. https:// doi.org/10.1016/j.copsyc.2018.12.005

Tiberius, V. y Hall, A. (2010). Normative theory and psychological research: Hedonism, eudaimonism and why it matters. The Journal of Positive Psychology, 5, 212-225. https://doi.org/10.1080/17439761003790971

Vago, D. R. y Silbersweig, D. A. (2012). Self-awareness, self-regulation, and self-transcendence (S-ART): a framework for understanding the neurobiological mechanisms of mindfulness. Frontiers in Human Neuroscience, 6, 296. https://doi.org/10.3389/fnhum.2012.00296

Van Gelder, J. L., Hershfield, H. E. y Nordgren, L. F. (2013). Vividness of the future self predicts delinquency. Psychological Science, 24(6), 974-980. https://doi.org/10.1177\%2F0956797612465197

Vázquez, C. y Hervás, C. (2008). Psicología Positiva aplicada. Desclee de Brower.

Wille, L., Van Hoye, G., Weijters, B., Rangarajan, D. y Carpentier, M. (2018). To be yourself or to be your ideal self? Outcomes of potential applicants' actual and ideal self-congruity perceptions. Journal of Personnel Psychology, 17(3), 107-119. https://doi.org/10.1027/1866-5888/a000213 